\title{
The Art of Radiology
}

\author{
Rochita Venkataramanan ${ }^{1}$ \\ ${ }^{1}$ Department of Clinical Radiology, Apollo Hospital, Chennai, India
}

J Gastrointestinal Abdominal Radiol ISGAR 2019;2:2-3

The curiosity of what lies within an object consumes everyone whether it is a child taking apart a toy or a surgeon performing a diagnostic laparotomy. Radiology is the branch of medicine that has been entrusted with taking pictures of deep structures within the body and laying open what is inside. Plain X-rays and various radiographic views provide a fascinating look at the skeleton and its diseases. These are easy to interpret as they look like dry bones held in the hand. However, in the realm of cross-sectional imaging, radiology spews out a profusion of peculiar pictures that require to be stacked up mentally to imagine the whole organ or disease as we see at dissection. Though radiologists are experts at such imagining of a whole structure such as the common bile duct or ureter from cross sections, physicians and surgeons find it very challenging to form such mental images. One surgeon friend mentioned to me that he finds these as gibberishly challenging as a series of software code ( - Fig. $\mathbf{1}$ ).

To help understand and analyze cross sections, threedimensional (3D) reconstructions of whole organs can now be performed by advanced computer software at workstations, especially with computed tomographic (CT) scans. These allow us to visualize the human body like never before. Sadly, these are often not done routinely, and the vast majority of cross-sectional reports are dispatched with just the standard axial images. Volumetric acquisition of scans that is key to these reconstructions is also not routinely performed.

The reason offered by most radiology departments for not providing detailed reconstructed images is that it is time consuming and technically difficult. Some departments in the West have 3D laboratories that have technologists generate and push these static images to the PACS for the radiologist to read. This laboratory is an addition to the radiology department cost causing concern. The static images are also vulnerable to artifacts that are created due to technique and do not offer an opportunity for real-time data manipulation and rotation of the image around the axis required. For example, the common bile duct cancer and its relationship

Address for correspondence Rochita Venkataramanan, MD, DNB, DMRD, Department of Clinical Radiology, Apollo Hospital, Chennai, India (e-mail: rochitav@yahoo.com; rochitav@gmail.com). to the hepatic arteries that are 2 to $3 \mathrm{~mm}$ in diameter, and sometimes smaller can be as-

sessed by making an oblique volumetric reconstruction along the plane of the duct and rotating it around its axis to bring the artery in view as well as doing the same for the artery. These complex manoeuvres are akin to an artist using the brush with dexterity bringing out on the canvas the image that the mind has formed ( - Fig. 2).

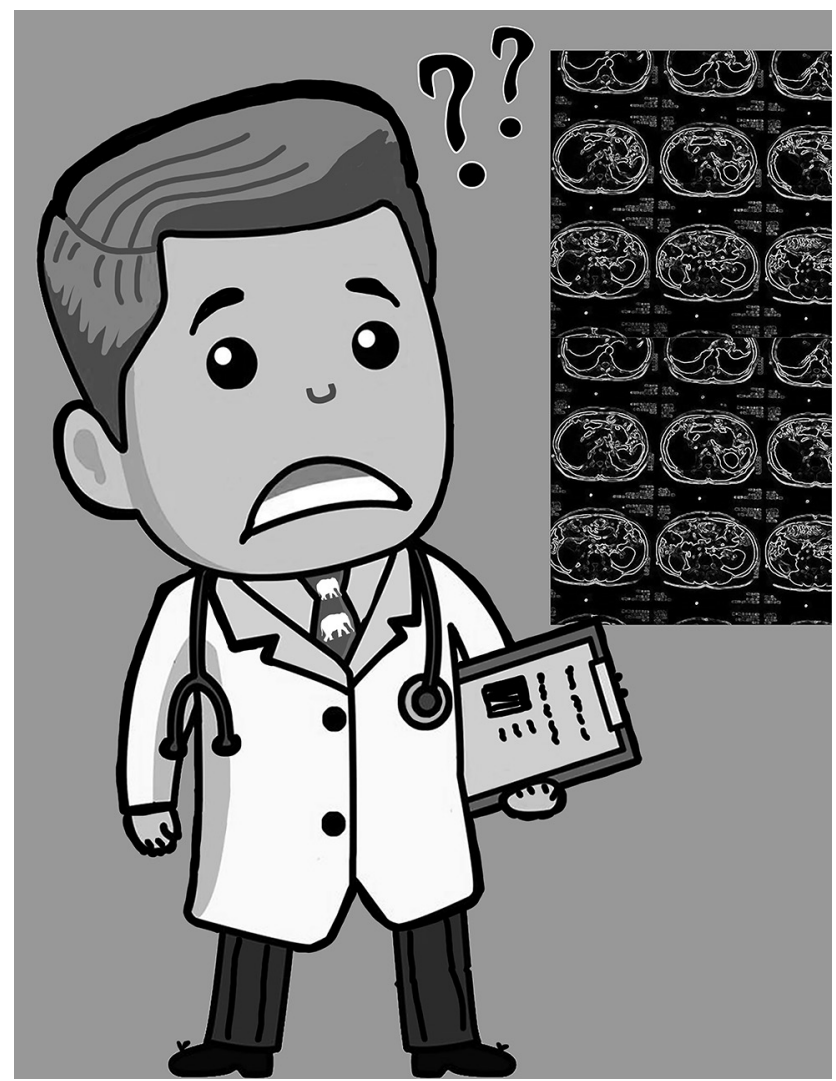

Fig. 1 Cartoon showing a clinician perplexed by innumerable axial sections through the abdomen.
Dol https://doi.org/ 10.1055/s-0039-1689685
(C)2019 Indian Society of Gastrointestinal and Abdominal Radiology
License terms

(ㄷ) (1) $\ominus \circledast$ 


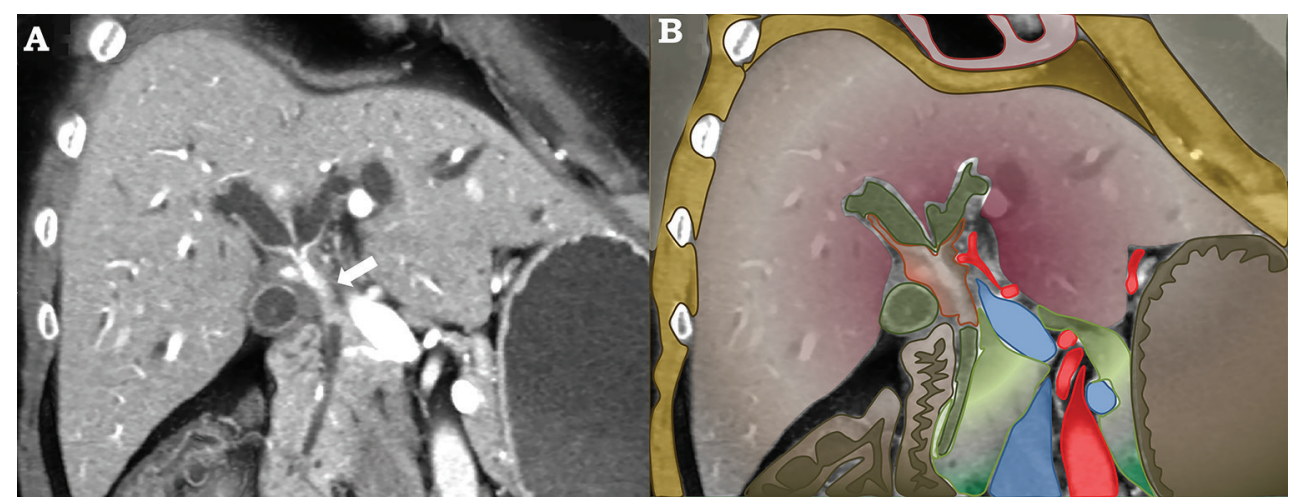

Fig. 2 Panel $\mathrm{A}$ is an oblique coronal reconstruction in the plane of the common bile duct showing its entire length and the cholangiocarcinoma (white arrow) involving the common hepatic duct. Panel $B$ is an artistic rendition of the same image as panel A.

Generation of 3D images requires an artistic disposition and eye. Today's radiologist is neither trained in this nor understands the value of this. These are useful not only for the referring clinician's use but also for the accuracy of image interpretation and final diagnosis.

Goodman and Kelleher ${ }^{1}$ in an interesting experiment connected radiology to art. They demonstrated that after a focused session of interpretation, training at a local art gallery where art experts taught novice radiology trainees how to thoroughly analyze a painting, first-year residents' ability to localize imaging abnormalities improved enormously.

There is significant emphasis on the way a radiology report should be written and much current debate about the pros and cons of a structured versus unstructured report. However, little literature exists on how cross-sectional radiology images should be generated to demonstrate the pathology. Representative imaging views and spot filming appear to be restricted to the two-dimensional X-ray and procedures such as barium studies for the gastrointestinal tract and the pyelo and cystograms for the urinary tract. As these studies become less and less utilized, the art of demonstration of pathology by a specific view seems to be dying.
Generation of representative images to demonstrate the findings in a radiology report is as important as the report itself. This is of great value especially in abdominal radiology where subtle gray shade differentiation is all one can rely on to separate disease from normal tissue. Images need to be in an interpretable format for a clinician who may not have an in-depth knowledge of radiology or the time required to study $0.50-\mathrm{mm}$-thin sections from the dome of diaphragm to pubic symphysis in arterial, early and late venous, and delayed phases that could yield a mind boggling 4,000 to 5,000 axial sections. The will and skill to produce one perfect masterpiece of a 3D picture that would sum up several lines in a written report and take one look to comprehend is what makes the science of radiology an art.

\section{Conflict of Interest}

None declared

\section{Reference}

1 Goodman TR, Kelleher M. Improving novice radiology trainees' perception using fine art. J Am Coll Radiol 2017;14(10):1337-1340 